\title{
Verificação da morfologia verbal em pré-escolares falantes do Português Brasileiro
}

\author{
Verification of verbal morphology in Brazilian Portuguese- \\ speaking preschoolers
}

\author{
Debora Maria Befi-Lopes ${ }^{1}$, Ana Manhani Cáceres²
}

\begin{abstract}
RESUMO
Objetivo: Este estudo buscou analisar quantitativamente o uso do tempo (presente, passado ou futuro), modo (indicativo, subjuntivo ou imperativo), número (singular ou plural) e pessoa ( $1^{\mathrm{a}}, 2^{\mathrm{a}}$ ou $3^{\mathrm{a}}$ ) dos verbos enunciados em situação de fala espontânea por préescolares falantes do Português Brasileiro, na cidade de São Paulo, em desenvolvimento normal de linguagem. Métodos: Coleta de amostras de fala de 60 pré-escolares divididos em três grupos pareados por gênero e faixa etária: GI (entre 2:0 e 2:11 anos), GII (entre 3:0 e 3:11 anos) e GIII (entre 4:0 e 4:11 anos). Resultados: A análise intragrupos demonstrou haver predominância do modo indicativo, do tempo presente, do número singular e da $3^{\text {a }}$ pessoa. A análise intergrupos indicou que o uso do modo indicativo é crescente, enquanto o imperativo é decrescente e o subjuntivo quase não ocorreu nesta amostra. O tempo presente não difere entre os grupos enquanto o passado e o futuro aumentam. Quanto ao número, o singular predomina, mas tanto singular como plural aumentam. Finalmente, a $3^{\mathrm{a}}$ pessoa predominou, a $2^{\mathrm{a}}$ decresceu e a $1^{\mathrm{a}}$ cresceu de GI para GIII. Conclusão: Os resultados demonstraram que os pré-escolares estudados aprimoraram o emprego da morfologia verbal ao longo de seu desenvolvimento, exibindo uma evolução gradual no domínio dos aspectos analisados. Por fim, não foram encontradas diferenças significantes na comparação dos gêneros.
\end{abstract}

Descritores: Desenvolvimento da linguagem; Linguagem infantil; Vocabulário; Pré-escolar

\section{INTRODUÇÃO}

Ao longo de seu desenvolvimento a criança, em um sentido estrito, apreende como uma língua - que será sua língua materna - relaciona sequências de sons vocais a uma entidade semântica; e, em um sentido mais amplo, ela desenvolve as habilidades de expressão e de interação social por meio desta língua, constituindo-se como sujeito da fala. É a este complexo processo que se atribui o termo aquisição da linguagem ${ }^{(1-2)}$.

Assim, o vocabulário e a cognição se desenvolvem conjuntamente a partir das interações sociais ${ }^{(3)}$. De fato, estudos relatam que, com o início do desenvolvimento da linguagem

Trabalho realizado no Laboratório de Investigação Fonoaudiológica em Desenvolvimento da Linguagem e suas Alterações do Departamento de Fisioterapia, Fonoaudiologia e Terapia Ocupacional da Faculdade de Medicina da Universidade de São Paulo - USP - São Paulo (SP), Brasil.

(1) Livre-Docente, Professora Associada do Departamento de Fisioterapia, Fonoaudiologia e Terapia Ocupacional da Faculdade de Medicina da Universidade de São Paulo - USP - São Paulo (SP), Brasil.

(2) Fonoaudióloga colaboradora do Laboratório de Investigação Fonoaudiológica em Desenvolvimento da Linguagem e suas Alterações do Departamento de Fisioterapia, Fonoaudiologia e Terapia Ocupacional da Faculdade de Medicina da Universidade de São Paulo - USP - São Paulo (SP), Brasil. Endereço para correspondência: Debora Maria Befi-Lopes. R. Cipotânea, 51, Cidade Universitária, São Paulo (SP), Brasil, CEP: 05360-000. E-mail: dmblopes@usp.br

Recebido em: 3/11/2008; Aceito em: 3/3/2009 ocorre uma ativação em determinadas regiões do hemisfério esquerdo, mais especificamente nos lobos temporal $\mathrm{e}$ frontal ${ }^{(4)}$. Além do que, vários aspectos do neurodesenvolvimento estão condicionados a aspectos biológicos, enquanto outros são fortalecidos pelas relações sociais e pelo meio em que estão inseridos ${ }^{(5)}$.

Desta forma, é possível supor que, a partir da imitação do que escuta, a criança possa aprender expressões linguísticas concretas, indicando que a linguagem é adquirida por meio do uso. Logo, este modelo ressalta a importância que a cultura imprime na aquisição e no desenvolvimento da linguagem ${ }^{(6)}$.

Estas informações sugerem, portanto, que a frequência de determinado estímulo interfere neste processo. Logo, as crianças não apenas dizem o que escutam, mas quanto mais escutam certa construção, mais lhes parece ser esta a única forma dela ser dita.

Um estudo acerca do aproveitamento das pistas do gênero gramatical, fornecidas pelo artigo, na compreensão de crianças falantes do Espanhol demonstrou que, entre o segundo e o terceiro anos de vida, elas já identificam a marcação de gênero para encontrar o referente de forma mais hábil. Outro fato interessante identificado, foi que aquelas que obtiveram um melhor desempenho, possuíam vocabulários mais amplos e suas produções demonstravam maior complexidade gramatical ${ }^{(7)}$.

Ao comparar a aquisição de verbos e substantivos ao lon- 
go do desenvolvimento lexical inicial, sugere-se que quando uma criança possui entre 50 e 100 palavras, o repertório de verbos começa a aumentar, estendendo-se até o momento em que a criança possui cerca de 500 palavras. Neste momento, ocorreria o balanceamento entre a proporção de verbos e demais palavras ${ }^{(8)}$.

O verbo consiste em uma palavra cujo referente não é tão claro quanto o do substantivo; sua aquisição costuma ocorrer de forma mais gradual. A ele é atribuída a superação dos enunciados de uma palavra para as combinações de diversas, o que favorece o desenvolvimento gramatical ${ }^{(9-10)}$. Esta aquisição não é simples; é apenas entre o terceiro e o quarto anos de vida, que as crianças aperfeiçoam o seu uso ${ }^{(6)}$.

Com relação à gramática, um estudo comparativo entre o Inglês e o Italiano, apontou que, tanto a idade cronológica quanto o tamanho do vocabulário, são indicadores efetivos do desenvolvimento linguístico; porém, o tamanho do vocabulário mostrou-se como a medida mais confiável, mesmo estando estes dois aspectos da linguagem intrinsecamente relacionados ${ }^{(11-14)}$.

Apenas a partir dos seis e sete anos, as crianças são hábeis para utilizar seus conhecimentos lingüísticos e determinar a transitividade de um verbo quando este é ambíguo, conforme demonstrou o estudo com crianças falantes do Inglês ${ }^{(15)}$. Crianças aprendendo Sesotho (uma das línguas Bantu), aos oito anos, ainda não possuem um desempenho equivalente ao de adultos, com relação ao conhecimento sobre a ordenação específica das palavras nesta língua, sugerindo que a evolução seja baseada na comparação de um item com o outro, para que se possa depois desenvolver as generalizações sintáticas ${ }^{(16)}$.

No Hebreu constatou-se que, inicialmente, o tempo verbal é empregado apenas para definir o aspecto do verbo; assim, sugere-se haver uma organização cronológica relacionada com o uso do tempo verbal e a aquisição da morfologia verbal, pois o gênero e o número foram empregados antes da pessoa ${ }^{(17)}$.

Um estudo da morfologia da flexão verbal na produção de crianças aprendizes do Espanhol constatou que a generalização começa a ocorrer após um período de performance adequada, e que os erros decorrentes diminuem com o passar dos anos; ou seja, o padrão descrito é compatível com o da curva em U. Logo, propõe-se que estes achados sejam compatíveis com um mecanismo duplo no qual o aprendizado da complexidade da morfologia verbal seria adquirido baseado na regra, para os verbos regulares e baseado na memória, para os verbos irregulares ${ }^{(18)}$.

No Português Brasileiro, é entre dois anos e dois anos e meio que as crianças geralmente começam a utilizar, com certa propriedade, os verbos em seus enunciados ${ }^{(19)}$. Nesta língua, um verbo nunca pode ocorrer sem ser flexionado e o tempo verbal sempre concorda com seu sujeito quanto à pessoa $\mathrm{e}$ ao número.

Com relação à flexão verbal, é importante mencionar que o tempo demonstra a situação do processo verbal em relação ao momento temporal em que se fala, isto é, o presente denota que o ato verbal ocorre no momento em que se fala; o passado denota que o ato verbal ocorre antes do momento em que se fala; e o futuro denota que o ato verbal ocorrerá após o momento em que se fala.
O modo indicativo serve para expressar ações definidas; o imperativo exprime ordens, além de expressar outros sentimentos, intenções e interesses; já o subjuntivo se caracteriza por expressar ações e fatos hipotéticos, isto é, tudo aquilo que está no campo de nossos desejos, de nossas aspirações.

O número indica se o verbo está sendo conjugado no singular ou no plural. A primeira pessoa verbal (eu, nós) designa quem fala; a segunda (tu, vós, você, vocês) com quem se fala; e a terceira (ele, ela, eles, elas) de que ou de quem se fala. Dois pontos importantes que merecem destaque são: (1) no Português Brasileiro, o pronome de tratamento você tem sido empregado no lugar do pronome reto $t u$ - segunda pessoa; assim, mesmo que desempenhe o papel de $2^{\mathrm{a}}$ pessoa do discurso, com quem se fala, por ser pronome de tratamento, gramaticalmente exige o verbo em $3^{\mathrm{a}}$ pessoa; e, (2) mais recentemente, tem ocorrido a substituição do pronome de $1^{\mathrm{a}}$ pessoa do plural nós pela expressão a gente. Tais fatos imprimem, portanto, mudanças no paradigma flexional verbal desta língua ${ }^{(20)}$.

Em uma pesquisa a respeito do uso da morfologia verbal em uma criança falante do Português Brasileiro, constatou-se haver forte relação entre a frequência no estímulo de certas formas verbais e seu uso pela criança. Além do que, a frequência da flexão verbal para o plural e para o singular é bastante diferente, sugestivo de que a concordância verbal é adquirida pouco a pouco, e que o aprendizado de flexões verbais específicas seja adquirido em um processo gradual $^{(21)}$.

Ainda, nesta língua, foi verificado que pré-escolares utilizam mais verbos que substantivos desde o segundo ano de vida. Dentre os verbos enunciados o tipo mais frequente é o intransitivo, enquanto o segundo mais frequente nas crianças com dois e três anos é o verbo de ligação; e nas crianças de quatro anos é o transitivo direto, sem diferenças significantes entre os gêneros ${ }^{(22)}$.

A partir do exposto na literatura, o presente estudo tem como objetivo verificar o uso do tempo (presente, passado ou futuro), modo (indicativo, subjuntivo ou imperativo), número (singular ou plural) e pessoa $\left(1^{\mathrm{a}}, 2^{\mathrm{a}}\right.$ ou $\left.3^{\mathrm{a}}\right)$ dos verbos enunciados por pré-escolares falantes do Português Brasileiro que residem na cidade de São Paulo.

\section{MÉTODOS}

Todos os sujeitos estavam matriculados em creches do município de São Paulo, localizadas na Zona Norte da referida cidade. As instituições mantinham convênio com a prefeitura e atendiam crianças de classe econômica média-baixa e baixa. Com o objetivo de caracterizar o desempenho de linguagem destas, foi realizada uma triagem fonoaudiológica ${ }^{(23)}$ que verificava as habilidades de vocabulário, fonologia, produção e compreensão de sentenças. Após a realização da triagem, foram selecionadas 60 crianças, em idade pré-escolar, com desenvolvimento normal de linguagem.

Previamente à coleta de dados, os pais foram esclarecidos a respeito dos objetivos da pesquisa e da forma de obtenção dos dados para somente então assinarem o Termo de Consentimento Livre e Esclarecido.

Os sujeitos foram divididos em três grupos, conforme a faixa etária a qual pertenciam, observando que cada grupo 
deveria conter 20 sujeitos, sendo dez do sexo feminino e dez do masculino. O Grupo 1 (GI) comporta a faixa etária de 2:0 a 2:11 anos, com média de dois anos e cinco meses; o Grupo 2 (GII) comporta a faixa etária de 3:0 a 3:11 anos, com média de três anos e quatro meses; e o Grupo 3 (GIII) comporta a faixa etária de 4:0 a 4:11 anos, com média de quatro anos e quatro meses.

Ao considerar as faixas etárias estudadas e a necessidade de obtenção de fala espontânea, a amostra de fala foi coletada a partir de situação de interação lúdica com brinquedos, a saber: miniatura de fazenda com animais, meios de transporte, alimentos, utensílios de cozinha e dois bonecos.

Além dos brinquedos, foram utilizados uma filmadora VHS, fitas de vídeo (uma para cada sujeito), um gravador e fitas cassete (também uma para cada sujeito).

Para a coleta dos dados, a pesquisadora preparou uma sala, posicionou a filmadora sobre um tripé e o gravador cassete ao lado da criança e dispôs os brinquedos sobre um tapete. Todos os brinquedos foram dispostos da mesma maneira para todos os sujeitos, sendo que os utensílios de cozinha ficavam localizados à frente e no centro, os animais e fazendinha à direita e os meios de transporte e bonecos à esquerda.

Cada uma das crianças era solicitada a se retirar de sua sala de aula e, então, era levada para esta sala. A criança era encorajada a interagir com a pesquisadora por um período de 30 minutos, durante o qual se realizava a filmagem. Durante a interação, a pesquisadora realizou perguntas abertas com o objetivo de propiciar a melhor situação de iniciativa por parte da criança. Além disso, reduziu as perguntas fechadas que necessitassem de respostas pontuais como sim ou não. Ressalta-se que os brinquedos utilizados propiciaram uma situação de comunicação efetiva, eliciando comentários e relatos por parte das crianças, inclusive as mais novas. Cada situação foi filmada em VHS e gravada em fita cassete.

Após a coleta dos dados, foi realizada a transcrição das amostras de fala. Para cada transcrição, o mesmo procedimento foi adotado: primeiramente, a pesquisadora assistia toda a gravação sem realizar nenhuma anotação, para observar o desempenho geral da criança. Em um segundo momento, ela assistia a gravação realizando a transcrição. A amostra de fala foi dividida em segmentos (cada enunciado produzido pela criança), até que se chegasse ao número esperado de 100 segmentos $^{(24)}$. Quando este número foi atingido, o restante da transcrição foi desprezado não fazendo parte da análise. Para que se pudesse perceber e dividir corretamente cada segmento, pelo menos um dos critérios deveria ocorrer: mudança de assunto por parte da criança, mudança do foco de atenção da criança, ou interrupção do terapeuta. Outro critério adotado na transcrição foi utilização de segmentos completamente transcritos, nenhum com quebras. Todas as repetições exatas de segmentos foram incluídas. As disfluências, como as repetições de palavra, foram pontuadas uma única vez. Em poucos casos, quando as palavras foram produzidas para dar ênfase, contou-se cada ocorrência ${ }^{(25)}$.

Após a transcrição de todas as fitas, a pesquisadora procedeu ao levantamento dos dados, listando os verbos para ser realizada a verificação destes de acordo com os seguintes aspectos: tempo (presente, passado ou futuro), modo (indica- tivo, subjuntivo ou imperativo), número (singular ou plural) e pessoa $\left(1^{\mathrm{a}}, 2^{\mathrm{a}}\right.$ ou $\left.3^{\mathrm{a}}\right)$.

Este estudo foi autorizado pela Comissão de Ética para Análise de Projetos de Pesquisa da Diretoria Clínica do Hospital das Clínicas da Faculdade de Medicina da Universidade de São Paulo sob número 360/01.

Para a análise estatística dos resultados foram utilizados os seguintes testes: teste-t pareado e independente e a análise de variância (ANOVA) para as comparações entre os gêneros e os grupos para cada uma das variáveis, supondo-se igualdade de variância e distribuição normal. Para as múltiplas comparações foi utilizado o teste de Tukey. O nível de significância adotado foi de $5 \%$. Os resultados significantes foram assinalados com um asterisco.

\section{RESULTADOS}

\section{Análise intragrupos}

A Figura 1 evidencia que o tempo presente predominou, enquanto o passado e o futuro aumentaram de GI para GII, e de GII para GIII, sendo o passado o segundo tempo mais recorrente em todos os grupos.

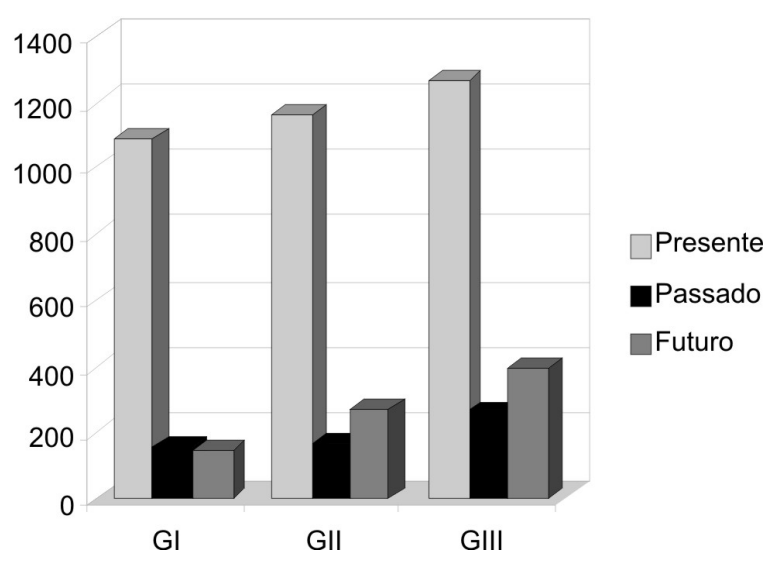

Figura 1. Classificação dos verbos de acordo com o tempo em cada grupo

Legenda: $\mathrm{GI}=$ grupo 1; GII = grupo 2; GIII = grupo 3

A Figura 2 demonstra que em relação ao modo há predominância e ampliação do indicativo em todos os grupos, sendo seguido pelo imperativo, que demonstrou decréscimo de ocorrência de GI para GII e deste para GIII. O subjuntivo ocorreu apenas em GIII e com valores mínimos.

Quanto ao número, a Figura 3 evidencia que o uso do singular e do plural aumentou de um grupo para outro, sendo, porém, o primeiro a predominar em todos os grupos.

Com relação à pessoa, observamos na Figura $4 \mathrm{a}$ ampliação na ocorrência da $3^{\mathrm{a}}$ pessoa, bem como sua predominância nos três grupos; a $1^{\mathrm{a}} \mathrm{e} 2^{\mathrm{a}}$ demonstraram comportamentos inversos: enquanto a primeira sofreu um acréscimo em sua ocorrência de um grupo para o outro, a outra decresceu de um para o outro - sendo que em GII apresentam valores bastante próximos.

Os resultados da ANOVA confirmam o predomínio do presente $(\mathrm{p}<0,001)$, do indicativo $(\mathrm{p}<0,001)$, do singular $(p<0,001)$, e da $3^{a}$ pessoa $(p<0,001)$. 


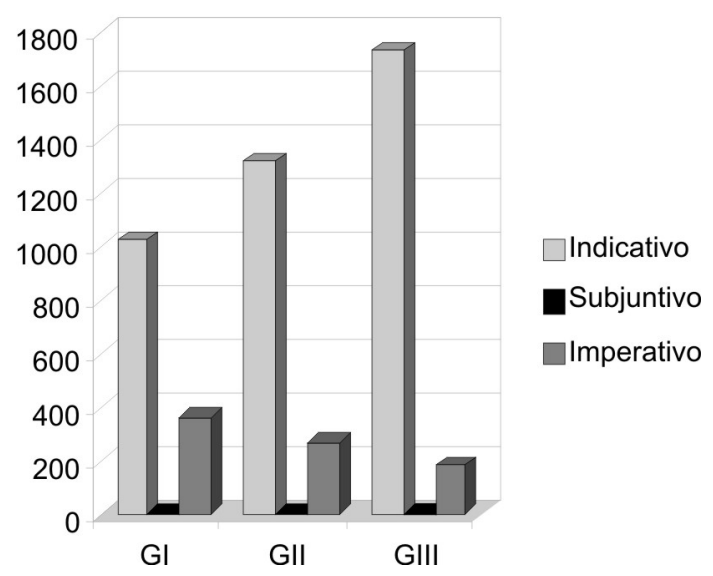

Figura 2. Classificação dos verbos de acordo com o modo em cada grupo

Legenda: $\mathrm{GI}=$ grupo 1; GII = grupo 2; GIII = grupo 3

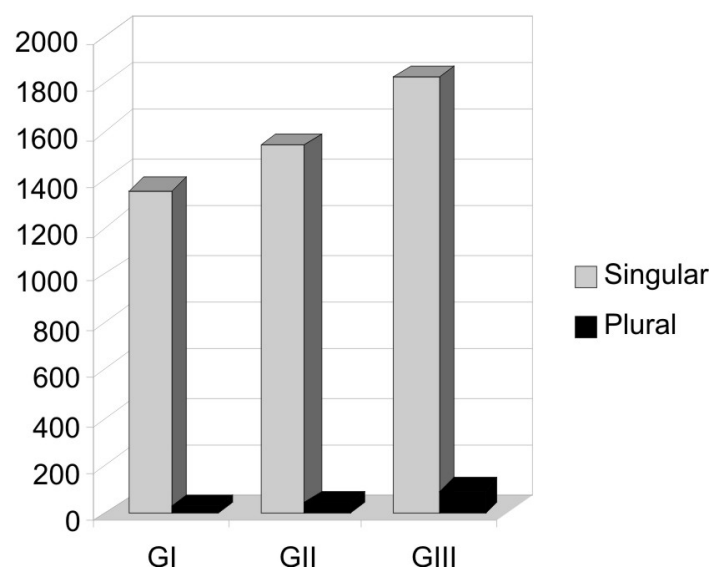

Figura 3. Classificação dos verbos de acordo com o número em cada grupo

Legenda: $\mathrm{GI}=$ grupo 1; GII = grupo 2; GIII = grupo 3

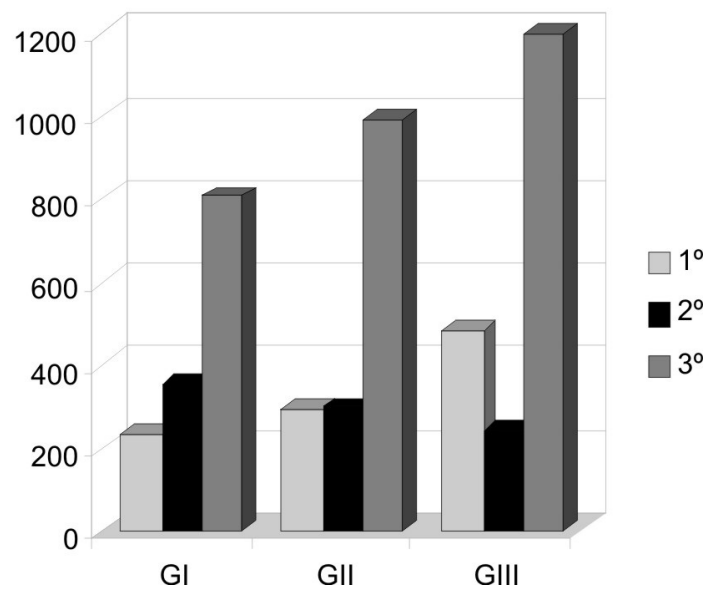

Figura 4. Classificação dos verbos de acordo com a pessoa em cada grupo

Legenda: $\mathrm{GI}=$ grupo 1; GII = grupo 2; GIII = grupo 3

\section{Análise intergrupos}

A análise intergrupos, com base nos testes de Tukey, revela que o uso de verbos no indicativo difere em todos os grupos, sendo GI o de menor ocorrência e GIII o de maior ocorrência. Quanto ao uso do subjuntivo os grupos não diferiram; já para o uso do imperativo os participantes de GI apresentaram maior ocorrência, seguido de GII e de GIII, mas a diferença foi estatisticamente significante apenas entre os extremos.

Quanto ao tempo verbal o uso de verbos no presente não difere nos grupos, mas o passado teve maior ocorrência em GIII. Já para o uso do futuro, todos os grupos diferem entre si, sendo GI o de menor ocorrência e GIII o de maior ocorrência.

A comparação relativa ao número verbal evidencia que para o uso tanto do singular quanto do plural há maior ocorrência em GIII.

Finalmente, foi empreendida a comparação entre os gêneros com o intuito de verificar a existência ou não de diferenças significantes. De maneira geral, os gêneros não diferiram, houve apenas três variações isoladas: em GIII, o gênero masculino produziu mais verbos no imperativo que $o$ feminino ( $\mathrm{p}=0,027$ ); em GIII, o gênero feminino produziu mais verbos no passado que o masculino $(\mathrm{p}=0,01)$; e, em GIII, o gênero feminino produziu mais verbos na terceira pessoa que o masculino $(\mathrm{p}=0,019)$.

\section{DISCUSSÃO}

Combinando as informações verificadas na literatura com a análise estatística dos achados são discutidos os resultados desta pesquisa.

Com relação ao modo verbal, nota-se que a evolução do indicativo é crescente de GI para GIII, e a do imperativo decrescente de GI para GIII - havendo diferença estatisticamente significante somente entre os extremos. Quanto ao uso do subjuntivo os grupos não se diferenciam, pois sua ocorrência é quase nula.

Considerando que o indicativo expressa ações definidas, o imperativo exprime ordens e sentimentos, e o subjuntivo se caracteriza por expressar hipóteses, podemos sugerir que por ser a linguagem um processo gradual e contínuo, seria preciso um tempo maior de experiência para que se pudesse dominar elementos que exigem refinamento não só linguístico, mas também cognitivo, como seria o caso do modo subjun-

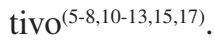

Com relação ao tempo verbal, há um predomínio do uso de verbos no presente que se mantém ao longo dos grupos; porém, o passado apresenta maior ocorrência no GIII e o futuro apresenta crescimento de GI para GIII.

Sabendo que o tempo indica a relação de um evento com o momento em que se fala, o presente equivale à ocorrência deste simultaneamente com a fala, ao passo que o passado equivale a este precedendo a fala, e o futuro equivale à fala que precede este. Em fases iniciais da vida, as crianças apropriam-se mais do presente, visto que a ação ou estado enunciado estão mais palpáveis e, gradualmente, vão tornando-se hábeis em enunciar fatos que já ocorreram ou irão ocorrer, demonstrando uma abstração mais acurada. Esta observação é condizente com o modelo de uso-baseado ${ }^{(5-6)}$ que preconiza que a partir de suas habilidades cognitivas, linguísticas e sociais o infante organiza e aperfeiçoa o conhecimento que adquire e o expressa verbalmente. 
Quanto ao número verbal, para todos os grupos predomina o uso de verbos no singular, e quanto à sua evolução verificamos um crescimento tanto para o singular quanto para o plural, alcançando seu ápice no GIII. Estes achados são compatíveis com os do estudo citado ${ }^{(17)}$, no qual indicam que a frequência no estímulo influi na produção da criança e que, portanto, este processo seria gradual.

Em relação à pessoa verbal, o uso da $3^{a}$ pessoa é predominante e, em GI e GII, a ocorrência da $1^{\mathrm{a}}$ e da $2^{\mathrm{a}}$ pessoa é igual; mas em GIII esta ocorrência é estatisticamente diferente, ou seja, a $1^{\mathrm{a}}$ pessoa é mais recorrente que a $2^{\mathrm{a}}$ pessoa. Considerando que a $1^{\mathrm{a}}$ pessoa verbal indica quem fala, a $2^{\mathrm{a}} \mathrm{com}$ quem se fala e a $3^{a}$ de que ou de quem se fala, podemos supor que o uso mais recorrente da $3^{\text {a }}$ deva-se ao fato de a situação de fala aqui utilizada, por envolver um objeto que intermedia a relação dos falantes, uma vez que geralmente é assim que se dão as situações de comunicação na infância, devido à necessidade de um referente concreto ${ }^{(13,15,17)}$, possa ter propiciado a maior utilização da $3^{\text {a }}$ pessoa.

De forma bastante semelhante ao discutido com relação ao modo, ao tempo e ao número, devemos considerar a característica processual da linguagem e, mais especificamente, a organização cronológica requerida para a flexão verbal em todos os seus aspectos. Neste estudo, verificamos a instabilidade na morfologia verbal entre o segundo e o quarto ano de vida, atentando para a interdependência das variáveis, por exemplo, o decréscimo no uso do modo imperativo e da $2^{\mathrm{a}}$ pessoa de GI para GIII, sugerindo, portanto, que a criança atente inicialmente para as propriedades da língua para, posteriormente, reconhecer suas particularidades ${ }^{(13)}$.

Outro aspecto interessante consiste em notar que realmente, no Português Brasileiro, o pronome de tratamento você $\left(2^{\mathrm{a}}\right.$ pessoa) ocorre em todos os grupos e, apesar de apresentar um decréscimo do GI para o GIII, seu uso nestas faixas etárias confirma a ocorrência de mudanças no paradigma flexional verbal desta língua ${ }^{(16)}$.

\section{CONCLUSÃO}

Esta pesquisa demonstrou que os pré-escolares estudados usam verbos predominantemente com modo indicativo, tempo presente, número singular e $3^{\text {a }}$ pessoa; além do que, houve um aprimoramento no emprego da morfologia verbal ao longo de seu desenvolvimento, com evolução gradual no domínio da língua Portuguesa falada no Brasil.

Os resultados favorecem o conhecimento sobre aspectos importantes do desenvolvimento da linguagem, especificamente no que se refere ao domínio e utilização dos verbos. Propiciam também dados objetivos para comparação de achados em avaliação de linguagem de crianças com distúrbios da comunicação, tanto na comparação de aspectos quantitativos quanto qualitativos, principalmente no que se refere ao desenvolvimento gramatical, ainda tão pouco explorado em crianças com alterações de linguagem falantes do Português Brasileiro. Obviamente pesquisas que comparem os resultados aqui encontrados com o desempenho de crianças com alterações de linguagem, avaliadas sob a mesma condição, necessitam ser realizadas.

\section{AGRADECIMENTOS}

À FAPESP pela concessão e prorrogação da bolsa de Iniciação Científica (processo 2005/04131-6).

\begin{abstract}
Purpose: This study sought to quantitatively analyze the use of tense (present, past or future), mood (indicative, subjunctive or imperative), number (singular or plural) and person (first, second or third) of the verbs enunciated in spontaneous speech by Brazilian Portuguese-speaking preschoolers from the city of São Paulo with normal language development. Methods: Speech samples from 60 preschoolers divided into three groups, paired by gender and age: GI (between 2:0 and 2:11 years), GII (between 3:0 and 3:11 years) and GIII (between 4:0 and 4:11 years). Results: Within-groups analysis showed prevalence of the use of indicative mood, present tense, singular and third person. Between-groups analysis indicated that the use of the indicative mood increased with age, while imperative decreased and subjunctive almost did not occur in this sample. The present tense did not show differences between groups, while past and future increased. Regarding number, the use of singular prevailed, but both singular and plural increased with age. Finally, the use of third person was prevalent, and the use of second person decreased, while first person increased from GI to GIII. Conclusion: The results showed that the preschoolers studied perfected the use of verbal morphology during their development, showing a gradual evolution on the domain of the aspects analyzed. No statistically significant differences were found in gender comparisons.
\end{abstract}

Keywords: Language development; Child language; Vocabulary; Child, preschool

\title{
REFERÊNCIAS
}

1. Corrêa, LM. Aquisição da linguagem e problemas do desenvolvimento lingüístico. Rio de Janeiro: Loyola; 2006. Conciliando processamento lingüístico e teoria de língua no estudo da aquisição da linguagem; p. 21-78. 
2. Borges LC, Salomão NM. Aquisição da linguagem: considerações da perspectiva da interação social. Psicologia: Reflexão e Crítica. 2003;16(2):327-36.

3. Vigotski LS. Pensamento e linguagem. São Paulo: Martins Fontes; 2005.

4. Yuan W, Holland SK, Cecil KM, Dietrich KN, Wessel SD, Altaye M et al. The impact of early childhood lead exposure on brain organization: a functional magnetic resonance imaging study of language function. Pediatrics. 2006;118(3):971-7.

5. Navas AL. Neurodesenvolvimento e linguagem. In: Berlim C, Muszkat M, Miranda MC, organizadores. Neuropsicologia de desenvolvimento. São Paulo: Memnon; 2006. p. 93-105.

6. Tomasello M. The item-based nature of children's early syntactic development. Trends Cogn Sci. 2000;4(4):156-63.

7. Lew-Williams C, Fernald A. Young children learning Spanish make rapid use of grammatical gender in spoken word recognition. Psychol Sci. 2007;18(3):193-8.

8. Barrett M. Desenvolvimento lexical inicial. In: Fletcher P, Mcwhinney B, organizadores. Compêndio da linguagem da criança. Porto Alegre: Artes Médicas; 1997. p. 299-321.

9. Skipp A, Windfuhr KL, Conti-Ramsden G. Children's grammatical categories of verb and noun: a comparative look at children with specific language impairment (SLI) and normal language (NL). Int J Lang Comm Disord. 2002;37(3):253-71.

10. Tyler LK, Randall B, Stamatakis EA. Cortical differentiation for nouns and verbs depends on grammatical markers. J Cogn Neurosci. 2008;20(8):1381-9.

11. Devescovi A, Caselli MC, Marchione D, Pasqualetti P, Reilly J, Bates E. A crosslinguistic study of the relationship between grammar and lexical development. J Child Lang. 2005;32(4):759- 86.

12. McGregor KK, Sheng L, Smith B. The precocious two-year-old: status of the lexicon and links to the grammar. J Child Lang. 2005;32(3):56385 .

13. Conboy B, Thal DJ. Ties between the lexicon and grammar: crosssectional and longitudinal studies of bilingual toddlers. Child Dev. 2006;77(3):712-35.
14. Hurtado N, Marchman VA, Fernald A. Spoken word recognition by Latino children learning Spanish as their first language. J Child Lang. 2007;34(2):227-49.

15. Brooks PJ, Zizak O. Does preemption help children learn verb transitivity? J Child Lang. 2002;29(4):759-81.

16. Demuth K, Machobane M, Moloi F. Rules and construction effects in learning the argument structure of verbs. J Child Lang. 2003;30(4):797821.

17. Armon-Lotem S, Berman RA. The emergence of grammar: early verbs and beyond. J Child Lang. 2003;30(4):845-77.

18. Clahsen H, Aveledo F, Roca I. The development of regular and irregular verb inflection in Spanish child language. J Child Lang. 2002;29(3):591622.

19. Barbosa JB. O uso dos verbos no desenvolvimento da linguagem. Revista Virtual de Estudos da Linguagem - Revel [Internet]. 2005 [citado 2009 Out 12];3(5): [cerca de 18 p.]. Disponível em: http://www. revel.inf.br/site2007/_pdf/5/artigos/revel_5_o_uso_dos_verbos_no_ desenvolvimento_da_linguagem.pdf

20. Maroneze BO. A realização do sujeito no português brasileiro [Internet]. [citado 2007 Jul 27]. Disponível em: http://www.orbilat.com/Languages/ Portuguese-Brazilian/Studies/Subject_realization.htm

21. Rubino RB, Pine JM. Subject-verb agreement in Brazilian Portuguese: what low error rates hide. J Child Lang. 1998;25(1):35-59.

22. Befi-Lopes DM, Cáceres AM, Araújo K. Aquisição de verbos em préescolares falantes do português brasileiro. Rev CEFAC. 2007;9(4):44452.

23. Fluharty NB. Fluharty preschool speech and language screening tests. Austin (TX): Pro-Ed; 1978.

24. Brown R. A first language: the early stages. Cambridge: Harvard University Press; 1973.

25. Araújo K. Aspectos do desempenho gramatical de crianças pré-escolares em desenvolvimento normal de linguagem [dissertação]. São Paulo: Universidade de São Paulo; 2003. 